\title{
Osteoporosis Healthcare Disparities in Postmenopausal Women
}

\author{
Irene Hamrick, M.D., ' Qing Cao, M.D., ${ }^{3}$ Dorothy Agbafe-Mosley, M.D., ${ }^{2}$ and Doyle M. Cummings, PharmD ${ }^{3}$
}

\begin{abstract}
Background: Previous studies in referral populations have shown that fewer African American women complete dual-energy x-ray absorptiometry (DXA) screening and are prescribed medications for osteoporosis. This study examines if these disparities exist in primary care practices.

Methods: Of 4748 eligible women $\geq 60$ years of age in primary care practices, we randomly selected 500 African American and 500 Caucasian women. We compared the DXA screening referral rate and results, follow-up rate, and medication prescribing for low bone mineral density (BMD) between African American and Caucasian women and analyzed provider demographics. We used logistic regression analysis to control confounding variables, such as age and BMI.

Results: Among the initial 1000 women, only 29.8\% African American Women were referred to DXA compared to $38.4 \%$ Caucasian women $(p<0.05)$, and $20.8 \%$ African American vs. $27.0 \%$ Caucasian $(p<0.05)$ women completed the test. Among women with a diagnosis of osteoporosis, African Americans were less likely to receive medication $(79.6 \%$ vs. $89.2 \%, p<0.05)$, without a difference in follow-up visit pattern between races. Female providers were more likely to refer women for DXA $(27.7 \%)$ than male providers $(21.7 \%)(p=0.035)$, and this gender difference in referral was more pronounced for African American patients.

Conclusions: Not enough eligible women are being screened and treated for osteoporosis in primary care. Even fewer African American women receive DXA screenings and are treated for osteoporosis. Controlling for age and BMI attenuated but did not eliminate the difference. Female providers were more likely than male providers to refer women for DXA.
\end{abstract}

\section{Introduction}

$\mathbf{N}$ OT ENOUGH WOMEN ARE BEING SCREENED for Osteoporosis. The U.S. Surgeon General in 2004 reported that less than one fourth of eligible women are being screened, even after fractures. ${ }^{1}$ The number of minority women who receive appropriate screening is even lower. ${ }^{2}$ The 2002 Report by the Institute of Medicine (IOM), Unequal Treatment: Confronting Racial and Ethnic Disparities in Health Care, ${ }^{3}$ documented consistent research findings showing that disparities exist across a range of clinical settings-including public and private hospitals, teaching and nonteaching hospitals-and a number of areas-including cancer, cardiovascular diseases, and mental illness. The disparities are reported to be associated with worse outcomes. The IOM report calls for standardized data collection to understand and eliminate these disparities. Despite this report, limited research has addressed the potential for disparity in osteoporosis screening and treatment.

Osteoporosis is a leading risk factor for fractures. Fragility fractures have an increased incidence in women with low bone mineral density (BMD), ${ }^{4}$ and BMD testing of the femoral neck of the hip, using dual-energy x-ray absorptiometry (DXA), is the best predictor of hip fracture risk. ${ }^{5}$ Nelson et al. ${ }^{6}$ analyzed the literature for harms and benefits of screening and found a $40 \%-50 \%$ reduction in fracture risk when women with low bone density are treated with bisphosphonates. Cauley et al. ${ }^{7}$ showed that decreased BMD is associated with increased risk of fracture in both older black and white women. They reported that African American women have higher BMD and lower fracture risk than Caucasian women. Although two risk calculators, the Simple Calculated

\footnotetext{
${ }^{1}$ Department of Family Medicine, University of Wisconsin, Madison, Wisconsin.

${ }^{2}$ New-Hanover Community Health Center, Wilmington, North Carolina.

${ }^{3}$ Department of Family Medicine, East Carolina University, Greenville, North Carolina.
} 
Osteoporosis Risk Estimation (SCORE) ${ }^{8}$ and the Foundation for Osteoporosis Research and Education 10 year fracture risk calculator (FORE) ${ }^{9}$ both include race as a protective factor, all other risk calculators, including World Health Organization Fracture Risk Assessment Tool $\left(\right.$ FRAX $\left.^{\circledR}\right)$ do not consider race. Basic practice guidelines recommended by the National Osteoporosis Foundation (NOF) ${ }^{10}$ the American College of Obstetricians and Gynecologists (ACOG) ${ }_{11}^{11}$ the American College of Rheumatologists (ACR) ${ }_{1}^{12}$ and the United States Preventive Services Task Force (USPSTF) ${ }^{13}$ do not include race as a factor that should influence osteoporosis screening or management. The Surgeon General's Report states that osteoporosis and fragility fractures are often mistakenly viewed by both the public and healthcare practitioners as being a problem only for older white women. ${ }^{1}$ The USPSTF recommends that women aged $\geq 65$ be screened routinely for osteoporosis. Routine screening for women aged $<65$ should be done only in those whose estimated 10-year fracture risk is $\geq$ that of a 65-year-old woman without additional risk factors. The ACOG also recommends screening women who are postmenopausal and $<65$ years who have at least one risk factor for osteoporosis. ${ }^{11}$

The exact risk factors that should trigger screening in this younger age group are difficult to specify based on evidence. Lower body weight (weight $<70 \mathrm{~kg}$ ) is the single best predictor of low BMD. ${ }^{14,15}$ As detailed in the latest USPSTF report, ${ }^{16}$ other organizations have also provided osteoporosis screening recommendations, including the NOF, the World Health Organization (WHO), the American College of Physicians, and ACOG. Medicare and insurance companies pay for DXA screening every 2 years in postmenopausal women. Although the optimal screening interval for older white women has been questioned, ${ }^{17}$ most would agree that not enough women are being screened for osteoporosis. ${ }^{1}$

Once osteoporosis is diagnosed, treatment should be provided to all women regardless of race. Limited data are available on the relative percentage of African American and Caucasian patients screened and treated for osteoporosis or osteopenia in primary care settings, a major focus of this article. Our previous studies examined DXA data in an already referred population and showed $19.4 \%$ of the screened women were African American and 80.6\% were Caucasian, whereas the reference population was $46 \%$ African American. ${ }^{2}$ Furthermore, we demonstrated in this referral population that African American patients are not treated for osteoporosis with bisphosphonates or other drug therapies as much as are Caucasians $(62 \%$ vs. $83 \%) .{ }^{18}$ However, the patients in these studies were already referred for DXA and may not represent what happens in primary care practices. There is a dearth of information on bone density screening and treatment of osteoporosis in primary care clinics. Therefore, this study examined women $\geq 60$ years old from an academic primary care clinic to further explore the racial differences in DXA screening for low BMD and in the active drug treatment of established osteoporosis and osteopenia.

\section{Materials and Methods}

\section{Patient population}

From 1998 to 2009, we identified all women $\geq 60$ years who came to our university medical center primary care clinics, including Family Medicine, General Internal Medicine, and Gynecology. Of the 4748 women, $44.7 \%$ were African Americans. Because of the small numbers of other races $(2.6 \%)$, we compared only African American and Caucasian women $(52.8 \%)$. We selected women $\geq 60$ years old because of postmenopausal status, which allows coverage of osteoporosis screening under Medicare and insurance carriers and because it was the recommendation in place at the time of our review. We randomly selected to analyze in detail the records of 1000 women, stratified by race to have equal numbers of African American $(n=500)$ and Caucasian $(n=500)$ women (Fig. 1). Based on our power calculation, a sample size of 500 women in each racial group would provide approximately $90 \%$ power to detect a difference of approximately $10 \%$ between groups in DXA referral rate. We collected provider demographics, including race, gender, and time in practice, of 213 primary care providers.

\section{Data collected}

One trained research assistant abstracted the primary care medical record (Centricity, General Electric) to determine if there was a referral for DXA screening, the referral visit adherence by the patient, the DXA scan results, the follow-up visit rate after DXA screening, and any medication prescribing for low BMD. For a given woman, any DXA referral or osteoporosis medication prescription between 1998 and 2009 was counted. In the case of patients with more than one DXA reading, the lowest score was used for designation of osteoporosis. The most recent height and weight were collected from the electronic health record for calculation of body mass index (BMI). Smoking, alcohol, and steroid use were documented from various areas in the record. The medical record system had fields for prior or current use of alcohol and smoking, with quantification. If either were documented, they were counted as positive. Quantity was not recorded, as cutoffs for risk of use in osteoporosis have not been established. Provider race, gender, time in practice, and specialty were recorded for referral for DXA and prescribing osteoporosis medications.

The DXA machine used in this study was a General Electric LUNAR DPXIQ Model 2288. We used the T-score from each DXA report for analysis of BMD, which compares the patient's BMD to a normative database of young Caucasian women. Patients whose DXA showed low BMD by WHO guidelines (T-score $\leq-2.5$ is osteoporosis and $\leq-1.0$ to $\geq$ -2.4 is osteopenia) were indentified. ${ }^{19}$ The Institutional Review Board at East Carolina University approved our study.

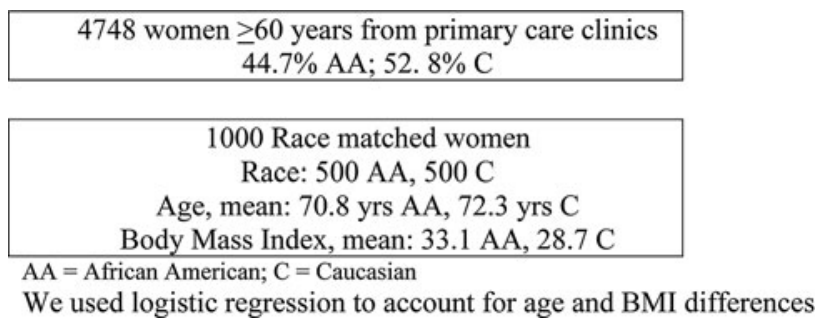

FIG. 1. Population flow chart. Logistic regression was used to account for age and body mass index differences. AA, African American; C, Caucasian. 


\section{Statistical analysis}

Chi-square and Fisher's exact tests were used to compare the proportion of women with each risk factor (e.g., smoking, alcohol use, glucocorticoid use), the proportion referred for DXA screening, adhering to the DXA screening appointment, and identified with a diagnosis of osteoporosis or osteopenia, as well as the proportion prescribed post-DXA medication treatment for osteoporosis or osteopenia by race. Chi-square analysis was also used to examine differences in referral and treatment patterns by provider demographic characteristics. To account for differences in age and BMI values in the two racial groups (Fig. 1), we constructed a series of logistic regression models to examine predictors of referral for DXA screening. All analyses were conducted using Statistical Package for the Social Sciences (SPSS) version 16 (SPSS, Inc., Chicago, IL).

\section{Results}

Osteoporosis risk factors, such as smoking, alcohol, and medications, did not differ between racial groups (Table 1). Among the 1000 women $(n=500$ African American and $n=500$ Caucasian) only $29.8 \%$ of African American women had been referred for DXA screening compared to $38.4 \%$ of Caucasian women $(p<0.05)$. Only a portion of these referred women were adherent with the DXA screening appointment, and this differed by race also (20.8\% African American vs. $27.0 \%$ Caucasian, $p<0.05$ ) (Table 2). About half as many African American as Caucasian women had a diagnosis of osteoporosis or osteopenia (low BMD) (Table 2). This number is greater than the DXA referral number, as it included identification of the diagnosis in the problem list by chart review. Some women may have been diagnosed on sustaining an insufficiency fracture or by prior DXA before joining our practice. Using regression analysis to control for age and BMI differences, we again found significantly fewer African American women referred for DXA (odds ratio [OR] 0.71, confidence interval [CI] 0.52-0.97). Age $\geq 65$ was significantly related to higher DXA referral rate than age $<65$ years (OR $2.8,95 \%$ CI 1.9-4.1).

Among those with a diagnosis of osteoporosis, African Americans were less likely to be prescribed medication for low BMD (79.6\% vs. $89.2 \%, p<0.05)$. Among those with a diagnosis of either osteoporosis or osteopenia, African American women were also less likely to be prescribed medication (mainly bisphosphonates) (Table 3).

Table 1. Racial Comparison of Risk Factors FOR OSTEOPOROSIS

\begin{tabular}{lcc}
\hline & \multicolumn{2}{c}{ Race matched women, $\mathrm{n}=1000(\%)$} \\
\cline { 2 - 3 } Population & $A A, \mathrm{n}=500(\%)$ & $\mathrm{C}, \mathrm{n}=500(\%)$ \\
\hline Glucocorticoid use & $114(22.8)$ & $95(19.0)$ \\
Smoking, previous & $88(17.6)$ & $106(21.2)$ \\
Smoking, current & $31(6.2)$ & $42(8.4)$ \\
Alcohol, previous & $314(62.8)$ & $30(6.0)$ \\
Alcohol, current & $29(5.8)$ & $76(15.2)$ \\
Age $\geq 65$ years & $337(67)$ & $367(73)$ \\
BMI $\leq 25$ & $112(22)$ & $204(41)$ \\
\hline
\end{tabular}

AA, African American; BMI, body mass index; C, Caucasian.
Table 2. Racial Comparison of Dual-Energy X-Ray Absorptiometry Referral and Completion RATES AND Rates of Diagnosis of Osteoporosis or Osteopenia

\begin{tabular}{lcc}
\hline & \multicolumn{2}{c}{ Race matched women, $\mathrm{n}=1000(\%)$} \\
\cline { 2 - 3 } Population & $A A, \mathrm{n}=500(\%)$ & $C, \mathrm{n}=500(\%)$ \\
\hline DXA referral, $n(\%)$ & $149(29.8)^{*}$ & $192(38.4)^{*}$ \\
DXA completion, $n(\%)$ & $104(20.8)^{* *}$ & $135(27.0)^{* *}$ \\
Osteoporosis, $n(\%)$ & $54(10.8)$ & $111(22.2)$ \\
Osteopenia, $n(\%)$ & $39(7.8)$ & $70(14.0)$ \\
\hline
\end{tabular}

Osteoporosis and osteopenia diagnosis rates are based on DXA results and chart review, which also includes women previously diagnosed from an earlier DXA or from a prior insufficiency fracture. ${ }^{*} p<0.01$

$* * p<0.05$.

We had reliable provider data for the entire sample and examined referral for DXA scanning as a function of provider demographics. Of the 213 providers, 158 (74\%) were Caucasian. We found male providers $(n=96)$ to refer fewer women for DXA than female providers $(n=117)(21.7 \%$ vs. $27.7 \%$, $p=0.035$ ), with an even greater difference for referral of African Americans women (15.4\% vs. $25.6 \%, p=0.007)$. No other provider demographics, including specialty, length of time in practice, or race, were significant.

In terms of healthcare use after screening, we did not find any significant difference by race in follow-up visit patterns for women with the diagnosis of osteoporosis who would have been considered candidates for treatment: African American $89.9 \%$ vs. Caucasian $83.9 \%(p=0.212)$.

\section{Discussion}

Our study showed that about one third of the eligible women in a primary care setting are being referred for osteoporosis screening or completed a DXA scan. Although this proportion is better than that described in the Surgeon General's report, ${ }^{1}$ it is clear that new strategies are needed to further improve the screening rate. Curtis ${ }^{20}$ has shown an

Table 3. Racial Difference in Medication Prescribed for Women $\geq 60$ Years Old with Diagnosis of Osteoporosis or Osteopenia

\begin{tabular}{lcc}
\hline & \multicolumn{2}{c}{$\begin{array}{l}\text { Women with Low BMD, } \\
\text { Model }\end{array}$} \\
\hline Women with low & $A A(\mathrm{n}=93 / 500)$ & $C(\mathrm{n}=181 / 500)$ \\
$\quad$ BMD by race & & \\
Low BMD, receiving & $73 / 93(78.5)^{*}$ & $159 / 181(88.0)^{*}$ \\
$\quad$ any medication, $n(\%)$ & & \\
Bisphosphonates & $38 / 93(41)$ & $80 / 181(44)$ \\
Teriparatide & $2 / 93(2.0)$ & $20 / 181(11)$ \\
Estrogen & $7 / 93(9.2)$ & $27 / 181(15)$ \\
Calcium & $26 / 93(28)$ & $73 / 181(40)$ \\
Vitamin D & $19 / 93(20)$ & $33 / 181(18)$ \\
Calcium/vitamin D & $1 / 93(1)$ & $7 / 181(4)$ \\
$\quad$ combination & & \\
\hline
\end{tabular}

${ }^{\mathrm{a}}$ Women with low BMD are based on DXA results and chart review; also includes women previously diagnosed from an earlier DXA or from a prior insufficiency fracture. ${ }^{*} p<0.05$. 
annual increase in DXA referral from $8.4 \%$ in 1999 to $12.9 \%$ in 2005 and a cumulative referral rate of $30 \%$ for women in that time interval. Screening may continue to improve in that more treatments are now available and at lower cost than in 2004 when the Surgeon General's report was published.

Despite guidelines for DXA screening that do not differentiate by race, our study underscores the continuing presence of racial disparities, with African American women from the same clinics being significantly less likely to be referred for a screening DXA scan than their Caucasian counterparts. This racial disparity is also true with regard to treatment with antiresorptive medications in patients with a diagnosis of osteoporosis or osteopenia. The IOM landmark report ${ }^{3}$ provides strong evidence that racial/ethnic disparities persist in medical care for a number of health conditions and services. The authors of the IOM report attribute disparities to three broad categories of decision making: (1) clinically appropriate differences, (2) environmental factors, such as insurance coverage and lack of access, and (3) discrimination based on biases, stereotyping, and uncertainty.

The first category of clinically appropriate differences may be reflected in the lower referral of African American women who, in our sample had higher BMI. The known higher BMD in African American women described in National Health and Nutrition Examination Survey (NHANES) data may influence provider behavior and may result in lower referral rates and prescribing of antiresorptive medications. However, treatment reduces fractures, ${ }^{6}$ and no evidence exists that treatment decisions based on clinical risk factors lead to better fracture outcomes than does BMD testing. Several screening tools have been developed that use known risk factors and calculate fracture risk as a percentage above which to treat: FRAX, ${ }^{21}$ Qfracture, ${ }^{22}$ Garvan, ${ }^{23}$ and FORE 10 year fracture risk calculator. ${ }^{9}$ These tools are free, provide no radiation exposure, and are accessible to patients, yet data comparing fracture and patient outcomes by race, based on a combined strategy that employs both DXA and these tools, are needed.

With regard to the second category, environmental factors, lack of access does not seem to be an issue. African American women had similar numbers of clinic visits as did Caucasian women. Although we did not have detailed insurance information on our patients, the large screening difference between those under and over 65 years of age was likely due to Medicare coverage for DXA screening after age 65. This, however, would not explain the racial difference, and unexplained differences still remain after these insurance factors are taken into account, suggesting the potential for disparity based on the third category of discrimination. When referral patterns were examined by provider gender, only male gender showed a lower referral pattern, and this was even stronger for African American women relative to Caucasian women. No information on the decision-making process of providers in our study is currently available to clarify the relative importance of these factors. However, in women aged $\geq 65$ years for whom routine DXA screening is recommended regardless of risk factor and among those with insurance, the disparity in screening and treatment rates for low BMD clearly persist.

African American referred women were less adherent with the DXA screening appointment. One reason for this difference may be explained by results of a qualitative study that showed that African American women do not feel at risk for osteoporosis. ${ }^{24}$ This pattern of racial difference in adherence to DXA screening appointments has also been shown for mammography screening. ${ }^{25}$

Another factor that may have influenced treatment patterns includes changes in recommendations from leading organizations. In the beginning of our study period, the WHO definitions for osteoporosis (T-score $\leq 2.5$ ) and osteopenia ( $\mathrm{T}$ score $\leq 1.0->2.5$ ) were used to define need for treatment. In 2003, the NOF changed recommendations for treatment to $\leq 2.0$ and $\leq 1.5$ with risk factors, and some providers may have changed their prescribing pattern for osteopenia patients, although this would not have affected the prescribing patterns for osteoporosis.

Strengths of our study include similar numbers of African American and Caucasian subjects, a detailed chart review that captured all relevant variables, use of regression modeling to address differences by race in risk factors, and provider information. Limitations of our study include the retrospective chart review design. The accuracy of this retrospective chart review study depended on the completeness of medical documentation, and it is possible that some providers did not thoroughly and completely document screening, treatment, or other variables collected. It is possible that some women may have taken hormone replacement therapy (HRT) in the early postmenopausal period, which may have influenced DXA referral. It is also possible that patients with a DXA referral but no results actually obtained a DXA scan at distant facilities. Among patients with a diagnosis and no drug treatment, we were unable to verify if these patients were also being treated by an outside physician.

This study involved African American and Caucasian women and cannot be generalized to other minority groups. The referral and treatment pattern found in our study in an academic center in the southeastern United States may also not represent physicians in private practice or in other areas of the country. Despite these limitations, our study identifies persistent racial disparity in osteoporosis healthcare among the postmenopausal women studied.

\section{Conclusions}

In our primary care population, significant unexplained racial disparities appear to exist in DXA screening referral and treatment of low BMD between African American and Caucasian women $\geq 60$ years who receive care in an academic primary care setting in the southeastern United States. Further research is indicated to investigate new strategies for increasing screening for low BMD to better understand the low DXA screening and treatment patterns in African American women and to identify specific interventions that address this racial disparity.

\section{Acknowledgments}

We thank Geoff McLeod, B.S., for data abstracting and analyzing and Mary Beth Plane, Ph.D., for review of the manuscript. I.H. received grant support through HRSA grant 1-K01 HP-00159-01 Geriatric Academic Career Award.

\section{Disclosure Statement}

No competing financial interests exist for all authors. 


\section{References}

1. Bone health and osteoporosis: A report of the Surgeon General. United States Department of Health and Human Services, 2004. Available at www.surgeongeneral.gov/ library/bonehealth/content.html. Accessed October 21, 2011.

2. Hamrick I, Steinweg KK, Cummings DM, Whetstone LM. Health care disparities in postmenopausal women referred for DXA screening. Fam Med 2006;38:265-269.

3. Smedley BD, Stith AY, Nelson AR, Institute of Medicine (U.S.). Committee on Understanding and Eliminating Racial and Ethnic Disparities in Health Care. Unequal treatment: confronting racial and ethnic disparities in health care. Washington, D.C.: National Academic Press; 2003.

4. Stone KL, Seeley DG, Lui LY, et al. BMD at multiple sites and risk of fracture of multiple types: Long-term results from the Study of Osteoporotic Fractures. J Bone Miner Res 2003;18:1947-1954.

5. Cummings SR, Black DM, Nevitt MC, et al. Bone density at various sites for prediction of hip fractures. The Study of Osteoporotic Fractures Research Group. Lancet 1993;341: 72-75.

6. Nelson HD, Helfand M, Woolf SH, Allan JD. Screening for postmenopausal osteoporosis: A review of the evidence for the U.S. Preventive Services Task Force. Ann Intern Med 2002;137:529-541.

7. Cauley JA, Lui LY, Ensrud KE, et al. Bone mineral density and the risk of incident nonspinal fractures in black and white women. JAMA 2005;293:2102-2108.

8. Cadarette SM, Jaglal SB, Murray TM. Validation of the simple calculated osteoporosis risk estimation (SCORE) for patient selection for bone densitometry. Osteoporos Int 1999;10:85-90.

9. 10-Year fracture risk calculator, 2010. Available at riskcalculator.fore.org./ Accessed October 21, 2012.

10. Why bone health is important, 2011. Available at www.nof .org/node/150 Accessed August 9, 2011.

11. Neff MJ. ACOG releases guidelines for clinical management of osteoporosis. Am Fam Physician 2004;69:1558-1560.

12. American College of Rheumatology position statement, 2008. Available at www.rheumatology.org/practice/clinical/ position/bonedensitymeasurement.pdf Accessed October 21, 2011.

13. Screening for osteoporosis, 2002. Available at www.ahcpr .gov/clinic/uspstf/uspsoste.htm Accessed July 21, 2010

14. Cadarette SM, Jaglal SB, Murray TM, McIsaac WJ, Joseph L, Brown JP. Evaluation of decision rules for referring women for bone densitometry by dual-energy x-ray absorptiometry. JAMA 2001;286:57-63.

15. Cadarette SM, Jaglal SB, Kreiger N, McIsaac WJ, Darlington GA, Tu JV. Development and validation of the Osteoporosis Risk Assessment Instrument to facilitate selection of women for bone densitometry. CMAJ 2000;162:1289-1294.

16. U.S. Preventive Services Task Force. Screening for osteoporosis: U.S. Preventive Services Task Force recommendation statement. Ann Intern Med 2011;154:356-364.

17. Gourlay ML, Fine JP, Preisser JS, et al. Bone density testing interval and transition to osteoporosis in older women. $\mathrm{N}$ Engl J Med 2012;366:225-233.

18. Hamrick I, Whetstone LM, Cummings DM. Racial disparity in treatment of osteoporosis after diagnosis. Osteoporos Int 2006;17:1653-1658.

19. Assessment of fracture risk and its application to screening for postmenopausal osteoporosis. Report of a WHO Study Group. World Health Organ Tech Rep Ser 1994;843:1-129.

20. Curtis JR, Carbone L, Cheng $\mathrm{H}$, et al. Longitudinal trends in use of bone mass measurement among older Americans, 1999-2005. J Bone Miner Res 2008;23:1061-1067.

21. Kanis JA, Johnell O, Oden A, Johansson H, McCloskey E. FRAX and the assessment of fracture probability in men and women from the UK. Osteoporos Int 2008;19:385-397.

22. Hippisley-Cox J, Coupland C. Predicting risk of osteoporotic fracture in men and women in England and Wales: Prospective derivation and validation of QFractureScores. BMJ 2009;339:b4229.

23. Chang KP, Center JR, Nguyen TV, Eisman JA. Incidence of hip and other osteoporotic fractures in elderly men and women: Dubbo Osteoporosis Epidemiology Study. J Bone Miner Res 2004;19:532-536.

24. Unson CG, Siccion E, Gaztambide J, Gaztambide S, Mahoney Trella P, Prestwood K. Nonadherence and osteoporosis treatment preferences of older women: A qualitative study. J Womens Health 2003;12:1037-1045.

25. Rahman SM, Dignan MB, Shelton BJ. Factors influencing adherence to guidelines for screening mammography among women aged 40 years and older. Ethnicity Dis 2003;13: $477-484$.

Address correspondence to: Irene Hamrick, M.D. 1100 Delaplaine Court Madison, WI 53715

E-mail: ihamrick@wisc.edu 